\title{
Stroke as Initial Manifestation of a Fulminant C. perfringens Sepsis
}

\author{
B Hess, P Hitzenberger, W Grisold, J Finsterer
}

Can. J. Neurol. Sci. 2008; 35: 260-261

Infections with the ubiquitous organism Clostridium $(C$.) perfringens not only manifest in the gastro-intestinal tract or the site of a surgical or traumatic wound, but also in the central nervous system (CNS). ${ }^{1}$ Stroke, however, has not been reported as an early manifestation of a fulminant $C$. perfringens sepsis.

\section{CASE Report}

The patient is an 81 -year-old caucasian male, height $170 \mathrm{~cm}$, weight $75 \mathrm{~kg}$, with a history of steatosis hepatis after cholecystectomy at age 76 years and pancreatitis with cholestasis at age 81 years. During this episode were elevated serum creatine-kinase, and serum lactate hyperuricemia, hepatomegaly, and diverticulosis of the sigmoid colon were noted. The history was negative for trauma, malignancy, surgery, vaccination, or immunosuppression. Risk factors for stroke were diabetes mellitus and arterial hypertension. No coagulation abnormality had been detected. His regular medication included omeprazole, diclofenac, allopurinol, and glimepiride.

Four months after the pancreatitis, at 2 a.m., he suddenly experienced aching of the left ear and later on both ears. In the morning of the same day he also complained about aching of the lumbar spine. At 10 a.m. oto-rhino-laryngological investigation was normal and he was dismissed again. At 2 p.m. the same day he developed fever up to $39.3^{\circ} \mathrm{C}$ and he attended the emergency unit at 6 p.m. Therapy with diazepam, non-steroidal analgesics, and opioids was ineffective. When repeatedly taking venous blood it became hemolytic after each drawing.

When taking the patient's history on admission to the Department of Infectious Diseases at 10 p.m., he suddenly developed speech disturbance, weakness of the right upper and lower limb, and became somnolent, suggesting an acute stroke. A computed tomogram (CT) scan of the cerebrum, however, showed only periventricular hypodensities and cloddy calcifications of the basal ganglia bilaterally. Cerebral diffusionweighted-MRI revealed a hyperintensity within the left white matter with a corresponding hypointensity on the ADC sequences. The patient was transferred to the Neurology Department at 11 p.m. where he presented with coma, preserved brainstem reflexes, flaccid tetraplegia, but without abnormal spontaneous ocular movements. The NIH-score was 23 , the Barthel-index 0, the Ranking-scale 5, and the Glasgow Coma Scale 3 . Because of hemolysis, only blood cell counts were usable. The erythrocyte count was $2.96 / \mathrm{pl}$ (n: 4.-5.5/pl), the hemoglobin $10.0 \mathrm{~g} / \mathrm{dl}(\mathrm{n}: 13-17 \mathrm{~g} / \mathrm{dl})$ and the hematocrit $19.7 \%$ (n: $43-50 \%$ ). Because of non-determinable coagulation parameters an acute thrombolytic therapy with urokinase was inconceivable. Body temperature was elevated up to $38^{\circ} \mathrm{C}$. Blood cultures were repeatedly taken, but no blind antibiotic therapy was initiated. The patient died at 2 a.m. on the next day, eight hours after admission to the emergency unit from a fulminant septic shock, disseminated intravascular coagulation (DIC) syndrome, and hemolysis.

On external inspection at autopsy there was generalized cutaneous gas edema. Gas edema was also found in the brain, heart, liver, guts, mesenterium, spleen, and kidneys (foam organs). Brain section additionally revealed malacia in the left periventricular, parietal region, in accordance with the cerebral MRI, and petechial cerebral bleedings ubiquitously. Inspection of the gastrointestinal tract revealed an acute ulcerophlegmoneous diverticulitis. Numerous thrombi were found in the aorta, coronary arteries, and the basal verebral arteries. Histological work-up of the organs disclosed multiple holes within the parenchyma of the brain, heart, lung, liver, spleen, and kidney, being attributed to gas generation. There was thickening of the left ventricular myocardium of $15 \mathrm{~mm}$ and dilation of all cardiac cavities. Interestingly, the left ventricular apex was hypertrabeculated. All blood cultures taken intra vitam grew gram-positive rods, identified as $C$. perfringens and gramnegative rods, identified as $E$. coli. From swabs of the brain, heart, and spleen also $C$. perfringens and E. coli were isolated. Cerebral arteries did not show artherosclerosis, any clot, or any stenosis.

\section{Discussion}

Infection with $C$. perfringens either manifests exclusively as local infection or starts from a local infection and spreads via hematogenic dissemination to fatal fulminant sepsis with hemolysis, disseminated intra-vasal coagulation, multi-organ failure, and ultimately death. Infection of the CNS is rare and may also originate from a local wound or from $C$. perfringens

From the Neurological Department (BH, PH, WG), Kaiser Franz-Josef Spital; Krankenanstalt Rudolfstiftung (JF), Vienna, Austria.

Received July 11, 2007. Final Revisions Submitted December 18, 2007. Reprint requests to: J. Finsterer, Postfach 20, Vienna, Austria. 
sepsis. Among the most prevalent CNS manifestations of $\mathrm{C}$. perfringens infection is meningitis. ${ }^{2}$ Rarely, meningitis is restricted to the spine. ${ }^{1}$ In single cases $C$. perfringens infection may manifest as local or diffuse pneumocephalus. ${ }^{3}$ After head trauma $C$. perfringens infection may also manifest as fatal encephalitis with intractable cerebral edema. In a single case $C$. perfringens infection manifested as subdural empyema. ${ }^{4}$ Stroke has not been reported as a CNS manifestation of a $C$. perfringens infection but among four patients with enterocolitis from $C$. perfringens, three developed mesenteric vein thrombosis, suggesting hypercoagulability as a manifestation of $C$. perfringens sepsis. Hypercoagulability with DIC-syndrome was also reported in a patient with $C$. perfringens infection of the urogenital tract. ${ }^{4}$ In animal studies sublethal dosages of the epsilon-toxin of $C$. perfringens type B caused neuronal damage in the hypocampus by excessive glutamate release. ${ }^{5}$

Stroke in the presented patient may have been due to gas embolism, metastatic pyemic embolism, cardiac embolism, or due to DIC-syndrome. Hypercoagulability may be explained by the effects of the alpha-toxin, which is present in all strains of $C$. perfringens and not only causes mistrafficking of neutrophils, such that they do not enter the infected tissue and modulate the host's cell metabolism by activating the arachnoid acid cascade, but also causes vasoconstriction and platelet aggregation, possibly reducing the blood supply in the infected tissue. ${ }^{6}$ Whether pre-existing atherosclerosis from diabetes or arterial hypertension or hypovolemia had an additional pathogenetic effect, remains elusive. The reason why the patient became comatose and tetraplegic remains speculative. A brain stem infarction was excluded upon autopsy. Blood pressure drops were excluded upon normal blood pressure on the stroke unit. Most likely coma resulted from the developing meningitis/ encephalitis. An empiric antibiotic therapy was not given because the infectious nature of the condition was not obvious. That stroke was misinterpreted as gas-related necrosis of the parenchyma cannot be definitively excluded but is rather unlikely given the distinctly different appearance of the ischemic area from the area with parenchymatous gas holes on autopsy. Furthermore, there was a diffuse infiltration of the parenchyma and holes were diffusely distributed, making a focal necrosis from the infection implausible.

In the presented patient we regard diverticulitis as the most probable origin of the infection because the history was negative for recent surgery, trauma, immunosuppression, or malignancy and there was the concomitant infection with E. coli, which is a nosocomial bacterium of the colon. Acute toxic colitis was excluded since only those parts of the sigmoid were affected, which also showed diverticles. Whether previous pancreatitis was already due to a $C$. perfringens infection or a manifestation of a non-syndromic mitochondrial disorder, remains speculative. Arguments for a mitochondrial disorder are the association of pancreatitis, basal ganglia calcification, diabetes, hyperuricemia, steatosis hepatis with hepatomegaly, arterial hypertension, lactacidosis, thickening of the left ventricular myocardium in the absence of actual arterial hypertension or valve stenoses, and left ventricular noncompaction. Whether ear pain and dorsalgia were due to local pressure from gas edema also remains questionable. Hemolysis was attributed to the alpha-toxin of $C$. perfringens, which has been previously reported to cause hemolysis. ${ }^{6}$ Alphatoxin splits lecithin to phosphocholine and diglyceride, thus impairing the functional integrity of the erythrocyte membrane and resulting in the development of spherocytes and lastly hemolysis. E. coli sepsis could have contributed to hemolysis, although such intense hemolysis is rarely seen.

This case shows that stroke may be one of the initial manifestations of a $C$. perfringens sepsis, originating from diverticulitis. In such a case stroke may originate from gas embolism, bacterial embolism, cardiac embolism, or from the concomitant coagulopathy.

\section{REFERENCES}

1. Kristopaitis T, Jensen R, Gujrati M. Clostridium perfringens: a rare cause of postoperative spinal surgery meningitis. Surg Neurol. 1999;51:448-50.

2. Debast SB, van Rijswijk E, Jira PE, Meis JF. Fatal clostridium perfringens meningitis associated with insertion of a ventriculoperitoneal shunt. Eur J Clin Microbiol Infect Dis. 1993;12:7201.

3. Meschia JF, Bhat RK, Dwinnell B, MacKenzie T, Slogosky S, Schneck S. Clostridium perfringens subdural empyema and meningitis. Neurology. 1994;44:1357-8.

4. Goyal M, Sharma R, Berry M. Diffuse pneumocephalus due to meningitis: CT findings. Pediatr Radiol. 1996;26:278-9.

5. Elejalde Guerra JI, Elcuaz Viscarret R, Almaraz Ruiz De Eguilaz MJ, Melendez Gracia A. Fulminate sepsis caused by Clostridium perfringens of urologic origin. Arch Esp Urol. 2002;55:446-8.

6. Miyamoto O, Sumitani K, Nakamura T, Yamagami S, Miyata S, Itano $\mathrm{T}$, et al. Clostridium perfringens epsilon toxin causes excessive release of glutamate in the mouse hippocampus. FEMS Microbiol Lett. 2000;189:109-13. 